\title{
Advances in Vibroacoustics and Aeroacustics of Aerospace and Automotive Systems
}

\author{
Roberto Citarella $^{1, *}$ (i) and Luigi Federico ${ }^{2}$ \\ 1 Department of Industrial Engineering, University of Salerno, 84084 Fisciano, Italy \\ 2 Italian Aerospace Research Centre (C.I.R.A), via Maiorise snc, 81043 Capua, Italy; 1.federico@cira.it \\ * Correspondence: rcitarella@unisa.it
}

Received: 23 February 2018; Accepted: 27 February 2018; Published: 3 March 2018

\section{Introduction}

Computing sound, taking into account the dynamical phenomena leading to its generation and transmission through fluid, is a challenging issue, with several applications into different industrial sectors [1,2]. Automotive and aerospace industries, dealing with vehicles and their comfortability for passengers' transportation and payload preservation, constitute two natural frameworks in which these subjects acquire a prominent role.

In principle, aeroacoustics calculations can be addressed solving Navier-Stokes equations, exploiting direct numerical simulations. In practice, such approach is extremely time-consuming, leading to the introduction of the analogy-based approaches, traditionally ascribed to the field of computational aeroacoustics (CAA). With this kind of method, the near field aerodynamics is computed, giving velocity and pressure fluctuations used as acoustic source terms [3].

Aeroacoustic noise represents a boundary condition applied on the vehicle model, in applications of industrial interest. Recently, the interest in such a kind of calculations is grown, thanks to the enhanced computational power of nowadays computers. In [4], for instance, a turbulent model was used in the CFD calculations and the rear of a car model was optimized using genetic algorithm method. This is an example of the increasing interest in detailed aeroacoustics simulations at the design level in automobiles.

Alongside with aeroacoustics, vibration studies cover a crucial role in applications [5,6]. Several deterministic and statistical approaches exist, and each of them bears advantages and limitations. The challenge when using deterministic methods in case of complex application systems derive from the need for accurate vibrational response of the model. Validating each mode of a complex structure, when the frequency range of interest covers hundreds of natural frequencies is computationally expensive. In addition, in order to have an accurate response, the model cannot be oversimplified and this adds a significant cost in terms of computation. The advantage of using statistical methods derives from simpler requirements for validating each eigenfrequency, even if these approaches are less accurate for a specific solution with a clear tonal resonance.

For these reasons, hybrid methods are appealing, merging together the strength point of both deterministic and statistical simulation techniques. Lower frequencies-where the tonal resonances are significant-are calculated applying finite element methods, whereas for higher frequencies, a statistic energy approach can be chosen.

The idea of identifying noise paths in vehicle noise transmission using statistical energy analysis (SEA) is not new, being already established in 1980s by DeJong. In [7], DeJong created a SEA model of a passenger car and used it to identify the transmission paths of acoustic energy from the engine, tires, and floor to the interior of the vehicle, predicting the noise level of the passenger compartment. In recent years, this idea has been extended: Musser and coworker [8], for instance, simulated vehicle interior noise levels using SEA, validating the study with a full sized car. 
On the other hand, the finite element method has gained attention, as in [9] where a finite element structural acoustic model of a car has been made.

As an alternative to FEM, the boundary element method (BEM) can be adopted for noise emission calculation [10,11]. In [12], the numerical modelling of noise radiated by a car body, using the so-called acoustic transfer vector (ATVs) and modal acoustic transfer vector (MATVs) concepts, is presented.

Combination of the aeroacoustics and vibroacoustics simulation analysis lies at the core of the current applied research in transportation industries. Müller and coworkers [13] used fast multipole BEM (FMBEM) to characterize the exterior aeroacoustic loads of a vehicle, combined then to statistical energy analysis simulation. Cotoni and coworkers [14] used FEM combined to statistical energy analysis simulation.

Concerning the FMBEM for the exterior problem, there still remains the famous issue of the so-called irregular or spurious frequencies, especially in the mid-frequency domain in which there is a high modal density.

Wang and coworkers [15] calculated a car interior noise level by combining the LES (large eddy simulation) to FEM/BEM simulation, validating CFD results in a wind tunnel by $1 / 3$ rd size model.

In summary, numerous newer strategies have been proposed in recent years, circumventing some of the problems and sources of inefficiency found in the past. The aim of this special issue is to help to shed some light on current progress in applied research in the field of transportation, linking the results obtained in recent years with industrial development, in the pursuit towards attaining more and more comfortable and sustainable mobility.

\section{The Present Issue}

We are deeply honored to be the guest editors of this thematic issue, which includes original articles and reviews of interest for the scientific community working on these subjects, as well as for industrial $R \& D$ departments. We are particularly grateful to the several leaders in these areas who decided to honor us with their valuable contributions. We want to thank them and their collaborators for the effort they have done for publishing their works in this issue of Applied Sciences. We are equally thankful to many colleagues who have helped us evaluating and reviewing these manuscripts with substantial improvements. In particular, we appreciate the editor-in-chief of Applied Sciences for inviting us to edit this topic issue and the staff of the editorial office who supported the entire process.

The purpose of this special issue is to highlight the latest advances in vibroacoustics and aeroacoustics applied to aerospace and automotive industries. In proposing this wide topic, we wanted to give scientists and engineers the opportunity to publish their studies of current interest, both in the theoretical and experimental fields of vibration, sound generation, and radiation; and passive and active noise control approaches, which can be of interest for assessing vibroacoustics characteristics of aerospace and automotive vehicles. In particular, we have tried to include articles focusing on the introduction of new approaches and methodologies in the vibroacoustics and aeroacoustics fields.

This issue provides access to several articles encompassing the aforementioned themes.

In the first article [16], an innovative integrated design verification process is described, based on the bridging between a new semiempirical jet noise model and a hybrid finite-element method/statistical energy analysis (FEM/SEA) approach, for calculating the acceleration produced at the payload and equipment level within the structure of Vega-C Launcher, vibrating under the external acoustic forcing field. The result is a verification method allowing for accurate prediction of the vibroacoustics in the launcher interior, using limited computational resources and without resorting to computational fluid dynamics (CFD) data.

Another paper of this issue [17], estimated the internal aerodynamic noise due to valve flow in a simple constriction-expansion pipe (a pressure relief device), by combining the large eddy simulation technique with a wavenumber-frequency analysis, which made it possible to decompose the fluctuating pressure into the incompressible hydrodynamic pressure and compressible acoustic pressure. The results showed that the acoustic pressure fluctuations in a pipe could be separated 
from the incompressible ones. This made it possible to obtain accurate information about the acoustic power, which could be used to assess the likelihood of a piping system failure due to acoustic-induced vibration, along with information about the acoustic power spectrum of each acoustic mode, which could be used to facilitate the systematic mitigation of the potential acoustic-induced vibration in piping systems.

In [18], flow-induced vibrations and the sound radiation of flexible plate structures of different thickness mounted in a rigid plate were experimentally investigated. Therefore, flow properties and turbulent boundary layer parameters were determined through measurements with a hot-wire anemometer in an aeroacoustic wind tunnel. Furthermore, the excitation of the vibrating plate was examined by laser scanning vibrometry.

In [19], fully-resolved rotor-fuselage interactional aerodynamics was used as the forcing term in a model based on the Euler-Bernoulli equation, aiming to simulate helicopter tail-boom vibration. The model was based on linear beam analysis and captured the effect of the blade-passing as well as the effect of the changing force direction on the boom. Results for the tail-boom vibration served to demonstrate the strong effect of aerodynamics on tail-boom aeroelastic behavior.

In [20], the mechanism by which plasma actuators can control the cavity tone was explored; in particular, the amount of sound reduction ('control effect') produced by actuators of differing dimensions was measured and direct aeroacoustic simulations were performed. The simulations showed that longitudinal streamwise vortices are introduced in the incoming boundary by the actuators, and the vortices form rib structures in the cavity flow. These vortices distort and weaken the two-dimensional vortices responsible for producing the cavity tone, causing the tonal sound to be reduced.

In [21], the prediction and reduction of noise from subsonic jets through the reconstruction of turbulent fields from Reynolds-averaged Navier-Stokes (RANS) calculations are addressed. This approach, known as stochastic noise generation and radiation (SNGR), reconstructs the turbulent velocity fluctuations by RANS fields and calculates the source terms of vortex sound acoustic analogy. An active fluid injection technique, based on extractions from turbine and injections of high-pressure gas into the main stream of exhausts, has been proposed and finally assessed with the aim of reducing the jet-noise through the mixing and breaking of the turbulent eddies. The SNGR method is, therefore, suitable to be used for the early design phase of jet-noise reduction technologies.

In [22], an aerodynamic noise optimization was performed by a non-linear acoustics solver (NLAS) approach for acoustic calculation. With the use of Kriging surrogate model, a multi-objective optimization of the streamlined shape of high-speed trains was obtained, which takes the noise level in the far field and the drag of the whole train as the objectives. To efficiently construct the Kriging model, the cross validation approach has been adopted.

In [23], a vibro-acoustic numerical and experimental analysis was carried out for the chain cover of a low powered four-cylinder four-stroke diesel engine. By applying a methodology used in the acoustic optimization of new engine components, firstly a finite element model (FEM) of the engine was defined, then a vibration analysis was performed for the whole engine (modal analysis), and finally a forced response analysis was developed for the only chain cover (separated from the overall engine). A boundary element (BE) model of the only chain cover was realized to determine the chain cover noise emission, starting from the previously calculated structural vibrations. All the information thus obtained allowed the identification of those critical areas, in terms of noise generation, in which to undertake necessary improvements.

The paper [24] provides a summary of some common research works carried out by the authors concerning computational methods for the prediction of the responses in the frequency domain of general linear dissipative vibroacoustics (structural-acoustic) systems for liquid and gas in the low-frequency (LF) and medium-frequency (MF) domains, including uncertainty quantification (UQ) that plays an important role in the MF domain. An efficient reduced-order computational model (ROM) is constructed using a finite element discretization (FEM) for the structure and the internal 
acoustic fluid. The external acoustic fluid is treated using a symmetric boundary element method (BEM) in the frequency domain. All the required modeling aspects required for the analysis in the MF domain have been introduced, in particular the frequency-dependent damping phenomena and model uncertainties.

Conflicts of Interest: The authors declare no conflict of interest.

\section{References}

1. Fahy, J.; Gardonio, P. Sound and Structural Vibration, 2nd ed.; Academic Press: Cambridge, MA, USA, 2007.

2. Ohayon, R.; Soize, C. Advanced Computational Vibroacoustics; Reduced Order Models and Uncertainty Quantification; Cambridge University Press: Cambridge, UK, 2014.

3. Wagner, C.; Huttl, T.; Sagaut, P. Large-Eddy Simulation for Acoustics; Cambridge University Press: Cambridge, UK, 2007.

4. Beigmoradi, S.; Hajabdollahi, H.; Ramezani, A. Multi-objective aeroacoustic optimization of rear-end in a simplified car model by using hybrid Robust Parameter Design, Artificial Neural Networks and Genetic Algorithm methods. Comput. Fluids 2013, 90, 123-132. [CrossRef]

5. Siano, D.; Citarella, R. Elastic Multi Body Simulation of a Multi-Cylinder Engine. Open Mech. Eng. J. 2014, 8, 157-169. [CrossRef]

6. Armentani, E.; Sbarbati, F.; Perrella, M.; Citarella, R. Dynamic analysis of a car engine valve train system. Int. J. Veh. Noise Vib. 2016, 12, 229-240. [CrossRef]

7. DeJong, R. A Study of Vehicle Interior Noise Using Statistical Energy Analysis; SAE Technical Paper 850960; SAE: Warrendale, PA, USA, 15 May 1985.

8. Musser, C.; Manning, J.; Peng, G. Predicting Vehicle Interior Sound with Statistical Energy Analysis. Sound Vib. 2012, 46, 8-14.

9. Durand, J.F.; Soize, C.; Gagliardini, L. Structural-acoustic modeling of automotive vehicles in presence of uncertainties and experimental identification and validation. J. Acoust. Soc. Am. 2008, 124, 1513. [CrossRef] [PubMed]

10. Citarella, R.; Landi, M. Acoustic analysis of an exhaust manifold by Indirect Boundary Element Method. Open Mech. Eng. J. 2011, 5, 138-151. [CrossRef]

11. Armentani, E.; Trapani, R.; Citarella, R.; Parente, A.; Pirelli, M. FEM-BEM Numerical Procedure for Insertion Loss Assessment of an Engine Beauty Cover. Open Mech. Eng. J. 2013, 7, 27-34. [CrossRef]

12. Citarella, R.; Federico, L.; Cicatiello, A. Modal acoustic transfer vector approach in a FEM-BEM vibro-acoustic analysis. Eng. Anal. Bound. Elem. 2007, 31, 248-258. [CrossRef]

13. Müller, S.; Cotoni, V.; Connelly, T. Guidelines for Using Fast Multipole BEM to Calculate Automotive Exterior Acoustic Loads in SEA Models. SAE Int. J. Passeng. Cars Mech. Syst. 2009, 2, 1530-1537. [CrossRef]

14. Cotoni, V.; Shorter, P.J.; Langley, R.S. Numerical and experimental validation of a hybrid finite element-statistical energy analysis method. J. Acoust. Soc. Am. 2007, 122, 259-270. [CrossRef] [PubMed]

15. Wang, Y.; Zhen, X.; Wu, J.; Gu, Z.; Xiao, Z.; Yang, X. Hybrid CFD/FEM-BEM simulation of cabin aerodynamic noise for vehicles traveling at high speed. Sci. China Technol. Sci. 2013, 56, 1697-1708. [CrossRef]

16. Bianco, D.; Adamo, F.P.; Barbarino, M.; Vitiello, P.; Bartoccini, D.; Federico, L.; Citarella, R. Integrated Aero-Vibroacoustics: The Design Verification Process of Vega-C Launcher. Appl. Sci. 2018, 8, 88. [CrossRef]

17. Kim, K.; Ku, G.; Lee, S.; Park, S.; Cheong, C. Wavenumber-Frequency Analysis of Internal Aerodynamic Noise in Constriction-Expansion Pipe. Appl. Sci. 2017, 7, 1137. [CrossRef]

18. Osterziel, J.; Zenger, F.J.; Becker, S. Sound Radiation of Aerodynamically Excited Flat Plates into Cavities. Appl. Sci. 2017, 7, 1062. [CrossRef]

19. Batrakov, A.; Kusyumov, A.; Kusyumov, S.; Mikhailov, S.; Barakos, G.N. Simulation of Tail Boom Vibrations Using Main Rotor-Fuselage Computational Fluid Dynamics (CFD). Appl. Sci. 2017, 7, 918. [CrossRef]

20. Yokoyama, H.; Tanimoto, I.; Iida, A. Experimental Tests and Aeroacoustic Simulations of the Control of Cavity Tone by Plasma Actuators. Appl. Sci. 2017, 7, 790. [CrossRef]

21. Barbarino, M.; Ilsami, M.; Tuccillo, R.; Federico, L. Combined CFD-Stochastic Analysis of an Active Fluidic Injection System for Jet Noise Reduction. Appl. Sci. 2017, 7, 623. [CrossRef] 
22. Sun, Z.; Zhang, Y.; Yang, G. Surrogate Based Optimization of Aerodynamic Noise for Streamlined Shape of High Speed Trains. Appl. Sci. 2017, 7, 196. [CrossRef]

23. Armentani, E.; Sepe, R.; Parente, A.; Pirelli, M. Vibro-Acoustic Numerical Analysis for the Chain Cover of a Car Engine. Appl. Sci. 2017, 7, 610. [CrossRef]

24. Ohayon, R.; Soize, C. Computational Vibroacoustics in Low- and Medium- Frequency Bands: Damping, ROM, and UQ Modeling. Appl. Sci. 2017, 7, 586. [CrossRef] 